\title{
Heavy Quark Diffusion with Relativistic Langevin Dynamics in the Quark-Gluon Fluid
}

\author{
Yukinao Akamatsu, Tetsuo Hatsuda, and Tetsufumi Hirano \\ Department of Physics, The University of Tokyo, Tokyo 113-0033, Japan
}

(Dated: October 22, 2018)

\begin{abstract}
The relativistic diffusion process of heavy quarks is formulated on the basis of the relativistic Langevin equation in Itô discretization scheme. The drag force inside the quark-gluon plasma (QGP) is parametrized according to the formula for the strongly coupled plasma obtained by the AdS/CFT correspondence. The diffusion dynamics of charm and bottom quarks in QGP is described by combining the Langevin simulation under the background matter described by the relativistic hydrodynamics. Theoretical calculations of the nuclear modification factor $R_{\mathrm{AA}}$ and the elliptic flow $v_{2}$ for the single electrons from the charm and bottom decays are compared with the experimental data from the relativistic heavy ion collisions. The $R_{\mathrm{AA}}$ for electrons with large transverse momentum $\left(p_{\mathrm{T}}>3 \mathrm{GeV}\right)$ indicates that the drag force from the QGP is as strong as the AdS/CFT prediction.
\end{abstract}

PACS numbers: 24.85.+p, 05.40.Jc, 11.25.Tq

\section{INTRODUCTION}

The physics of the quark-gluon plasma (QGP) is actively studied by means of the relativistic heavy-ion collisions at Relativistic Heavy Ion Collider (RHIC) in BNL and will be pursued further at Large Hadron Collider (LHC) in CERN [1]. The space-time evolution of the heavy-ion collisions at RHIC is well described by the $(3+1)$-dimensional relativistic hydrodynamics supplemented with the hadronic cascade after chemical freezeout [2]. Information on the collective dynamics of QGP is obtained by the soft probes such as distributions of light hadrons at low momentum, while the information of microscopic dynamics of QGP is obtained by the hard probes such as jets, heavy quarks, and heavy quarkoniums [3].

In the present paper, we focus on charm and bottom quarks which behave as impurities in QGP. Experimentally, the signal of the heavy quarks can be extracted from the single electron spectra through semileptonic decays [4, 5]. Theoretically, the energy loss of heavy quarks in QGP has been estimated in perturbative QCD (pQCD) techniques [6, 7]. However, it was pointed out recently that the convergence of the weak coupling expansion of the drag force for heavy quarks is rather poor at the temperature relevant to RHIC and LHC, so that the calculation in the leading order would not be reliable [8]. Possible alternative way to estimate the drag force is to use the duality conjecture between the gauge theory and string theory (AdS/CFT correspondence) [9, 10, 11]. Although it can be applied only to the $\mathcal{N}=4$ supersymmetric Yang-Mills plasma with large 't Hooft coupling, the result obtained may provide us with a hint for the drag force in the strong coupling regime of the QCD plasma if appropriate translation is made [12].

The purpose of this paper is to study the connection between the drag force acting on the charm and bottom quarks in QGP and the final electron spectra. To make such connection, we introduce relativistic Langevin equation for heavy quarks under the background of the quark-gluon fluid described by the ideal hydrodynamics. We need relativity since the transverse momentum of the heavy quarks at RHIC is not necessarily smaller than their rest masses. Our relativistic Langevin equation is formulated in Itô discretization scheme. The diffusion constant and the drag force are related through a generalized fluctuation-dissipation relation. As for the drag force, two distinct models, pQCD and AdS/CFT, are considered. To calculate the space-time dynamics of light quarks and gluons, $(3+1)$-dimensional hydrodynamics is used, which is necessary to calculate the electron spectra of different impact parameters in the heavy ion collisions. The Langevin equation for heavy quarks is numerically solved from the initial distribution generated by Monte Carlo generator PYTHIA [13] until the freezeout of the heavy quarks. The transverse-momentum $\left(p_{\mathrm{T}}\right)$ dependence of the nuclear modification factor $\left(R_{\mathrm{AA}}\right)$ and the elliptic flow $\left(v_{2}\right)$ of single electrons as decay products of heavy quarks are calculated and compared with the RHIC data.

This paper is organized as follows. In Sec. III after formulating the relativistic Langevin equation and a generalized fluctuation-dissipation relation, we introduce two extreme models of the drag force motivated by pQCD and AdS/CFT. In Sec.III, the relativistic hydrodynamics for light degrees of freedom and the relativistic Langevin equation for heavy degrees of freedom are combined in order to describe the heavy quark diffusion in dynamical QGP fluid. The initial condition of heavy quarks, the algorithm of Langevin simulation in dynamical background, and the treatment of the freezeout and decay of heavy quarks are discussed in detail. In Sec. IV] the numerical results of our calculation are presented. We show the profile of heavy quark diffusion, heavy quark spectra, and single electron spectra and compare our single electron spectra with experimental data. Physical meaning of our results are also discussed. Section V is devoted to summary and concluding remarks. In Appendix A, 
we show a derivation of the relativistic Kramers equation from the relativistic Langevin equation in the Itô discretization scheme.

\section{LANGEVIN DYNAMICS OF HEAVY QUARKS}

In this section, we formulate the relativistic Langevin equation in the local rest frame of the fluid. A generalized form of the fluctuation-dissipation relation is derived. Then, we discuss the drag forces calculated in the pQCD approach and in the AdS/CFT approach. Finally, we introduce a phenomenological model of the drag coefficient $\Gamma$ and the diffusion constant $D$ which satisfy the generalized fluctuation-dissipation relation.

\section{A. Relativistic Brownian motion}

Suppose that there exist a time scale $\tau_{\mathrm{B}}$ during which a Brownian particle changes its momentum and a microscopic time scale $\tau_{\mathrm{m}}$ during which light dynamical degrees of freedom change state and lose time correlation. If these time scales satisfy $\tau_{\mathrm{B}} \gg \tau_{\mathrm{m}}$, one can describe the Brownian particle by the Langevin equation [14]. In such a situation that the charm/bottom diffuses inside the quark-gluon plasma, there are some extra complications: (i) The background quark-gluon fluid expands rapidly in space and time with the local 4 -velocity $u^{\mu}(\vec{x}, t)$, and (ii) the initial momentum distribution of the charm/bottom governed by the hard QCD process has high momentum component larger than their quark masses.

As for (i), we define the Langevin equation in the rest frame of matter $\left(u^{\mu}=(1,0,0,0)\right)$ and the real motion of the Brownian particle is obtained by the local Lorentz boost back to the moving frame. As for (ii), we take into account the relativistic kinematics of the Brownian particle in a minimal way by using relativistic dispersion relation $E(p)=\sqrt{p^{2}+M^{2}}$. Then the Langevin equation in the rest frame of matter with minimum relativistic kinematics may be written as 15]

$$
\begin{aligned}
\Delta \vec{x}(t) & =\frac{\vec{p}}{E(p)} \Delta t, \\
\Delta \vec{p}(t) & =-\Gamma(p) \vec{p} \Delta t+\vec{\xi}(t) .
\end{aligned}
$$

Here $\Delta \vec{x}(t)=\vec{x}\left(t^{\prime}\right)-\vec{x}(t)$, and $\Delta \vec{p}(t)=\vec{p}\left(t^{\prime}\right)-\vec{p}(t)$, and $\Delta t \equiv t^{\prime}-t$ is a discrete step of time. The momentum dependent drag coefficient is denoted by $\Gamma(p)$ which is related to the time scale of the Brownian particle $\tau_{\mathrm{B}} \sim \Gamma^{-1}$. Also $\vec{\xi}(t)$ is a noise obeying the probability distribution $W[\xi(t)]$ which we take to be the Gaussian white noise with a normalization constant $C$ :

$$
W[\vec{\xi}(t)] d^{3} \xi(t)=C \exp \left[-\frac{\vec{\xi}(t)^{2}}{2 D(p) \Delta t}\right] d^{3} \xi(t) .
$$

This leads to

$$
\begin{aligned}
\left\langle\xi_{i}(t)\right\rangle & =0, \\
\left\langle\xi_{i}(t) \xi_{j}\left(t^{\prime}\right)\right\rangle & =D(p) \delta_{i j} \delta_{t t^{\prime}} \Delta t,
\end{aligned}
$$

where $D(p)$ is a momentum dependent diffusion constant. Note that $\vec{\xi}(t)$ is not a single microscopic kick but a sum of microscopic kicks during the time $\Delta t$.

Throughout this paper, we use the Itô discretization scheme of the Langevin equation; namely, all the argument of $\vec{p}$ in the right hand side of Eqs. (10) and (2) are evaluated at the pre-point time $t$. This is particularly useful for numerical simulations due to obvious reason. The relativistic Kramers equation, which is a partial differential equation for the probability of the particle distribution in the phase space $P(\vec{p}, \vec{x}, t)$, is then obtained as (see the derivation in Appendix A)

$$
\begin{aligned}
& \left(\frac{\partial}{\partial t}+\frac{\vec{p}}{E} \frac{\partial}{\partial \vec{x}}\right) P(\vec{p}, \vec{x}, t) \\
& \quad=\frac{\partial}{\partial \vec{p}}\left(\Gamma(p) \vec{p}+\frac{1}{2} \frac{\partial}{\partial \vec{p}} D(p)\right) P(\vec{p}, \vec{x}, t) .
\end{aligned}
$$

Note that $\partial / \partial \vec{p}$ acts not only on $P$ but also on $\Gamma(p)$ and $D(p)$.

Demanding that Eq. (6) has the relativistic MaxwellBoltzmann distribution (the Jüttner distribution) $P(\vec{p}, \vec{x}, t) \propto \exp \left[-\sqrt{p^{2}+M^{2}} / T\right]$ as a stationary solution, we obtain a constraint between the drag and the diffusion as

$$
\Gamma(p)+G(p)=\frac{D(p)}{2 E T},
$$

with $G(p) \equiv d D(p) / 2 p d p=d D(p) / d\left(p^{2}\right)$. If $D$ is $p$ independent, Eq. (7) reduces to the relativistic analogue of the Einstein relation $\Gamma=\frac{D}{2 E T}=\frac{M}{E} \frac{D}{2 M T}$ obtained in [15].

\section{B. Modeling the energy loss of heavy quarks}

Energy loss of heavy quarks in the deconfined phase has two sources; the collisional energy loss due to elastic scattering of a heavy quark with the plasma constituents and the radiative energy loss associated with the induced emission of the gluon. In the leading order (LO) of the weak-coupling QCD perturbation, these processes are found to have different momentum dependence of the heavy quark and could become comparable in magnitude [6, 7]. Recently, the convergence of such weak-coupling expansion was questioned by an explicit calculation of the collisional process in the next-to-leading order (NLO) [8]: The drag coefficient for the 3-color, 3-flavor QCD in the non-relativistic kinematics $(M \gg T, p)$ reads

$$
\begin{aligned}
& \left.\Gamma_{\mathrm{pQCD}}\right|_{M \gg T, p} \\
& \quad \simeq \frac{8 \pi}{3} \alpha_{\mathrm{s}}^{2} \frac{T^{2}}{M}(-\ln g+0.07428+1.8869 g) .
\end{aligned}
$$


For the QCD coupling constant relevant at RHIC and LHC $(g \sim 2)$, the weak-coupling expansion has an obvious problem of convergence. From the phenomenological point of view, it has been argued in the past that relatively large drag force is necessary to account for the RHIC data [16, 17].

Alternative approach to the drag force is provided by the AdS/CFT correspondence [9, 10, 11]. In the $\mathcal{N}=4$ super-Yang-Mills theory (SYM) at large $N_{\mathrm{c}}$ and large 't Hooft coupling $\lambda \equiv g_{\text {SYM }}^{2} N_{\mathrm{c}}$, energy loss of an external quark with velocity $v$ is obtained as

$$
\begin{aligned}
\frac{d p}{d t} & =-\frac{\pi \sqrt{\lambda}}{2} T_{\mathrm{SYM}}^{2} \frac{v}{\sqrt{1-v^{2}}} \\
& \simeq-\frac{\pi \sqrt{\lambda}}{2} T_{\mathrm{SYM}}^{2} \frac{p}{M} .
\end{aligned}
$$

Here the first equation is valid for arbitrary mass of external quark, while the second equation is valid for $M \gg$ $\sqrt{\lambda} T[11]$. By matching the energy density and the heavy quark potential in the SYM plasma to those in QCD plasma, one finds $T_{\mathrm{SYM}} \simeq T_{\mathrm{QCD}} / 3^{1 / 4}$ and $3.5 \leq \lambda \leq 8.0$ [12]. Then, the drag coefficients may be estimated as

$$
\Gamma_{\mathrm{AdS} / \mathrm{CFT}}=(2.1 \pm 0.5) \frac{T^{2}}{M} .
$$

A remarkable feature of this formula in contrast to the weak-coupling estimate is that $\Gamma$ is $p$-independent 11 . The fundamental question here is, of course, the reliability of the translation from SYM to QCD both conceptually and numerically.

Given the theoretical uncertainties in estimating the drag force as mentioned above, we will take a phenomenological approach in this paper: We adopt the parametric dependence of the drag coefficient motivated by the AdS/CFT in Eq. (11) with the overall magnitude left as a free parameter:

$$
\Gamma \equiv \gamma \frac{T^{2}}{M}
$$

The dimensionless drag coefficient $\gamma$ is assumed to be independent of $T, M$, and $p$ throughout this paper. The corresponding diffusion constant $D$ is obtained from the generalized fluctuation-dissipation relation in Eq. (7) with the physical boundary condition, $D \rightarrow 0$ as $\Gamma \rightarrow 0$ :

$$
D=2 E T \cdot \Gamma \cdot\left(1+\frac{T}{E}\right)=\gamma \frac{2 T^{3}}{M}(E+T) .
$$

It is in order here to make two remarks on the dynamics we employed in Eqs. (12) and (13). (i) Since we have assumed $\Gamma$ to be $p$-independent motivated by AdS/CFT, the diffusion constant $D$ depends necessarily on the momentum of the Brownian particle. One may alternatively assume that $D$ is independent of $p$ while $\Gamma$ depends on $p$ as $\Gamma(p)=D /(2 E(p) T)$ [15]. Such dynamics would simulate the $p$-dependence of the drag force due to collisional process in the weak-coupling regime [7]. (ii)
At ultra-high energies $p \gg M$, the dominant energy loss occurs through the induced emission of the gluons. In this case, the condition $\tau_{\mathrm{B}} \gg \tau_{\mathrm{m}}$ is violated. Thus the Langevin approach becomes inapplicable 11] and a different approach based on radiative energy loss is required to describe heavy quarks in the QGP [7, 18]. With these reservations in mind, we consider our ansatz Eqs. (12) and (13) as phenomenological but characteristic dynamics of QCD and try to estimate $\gamma$ from the observed single electron data at RHIC in later sections.

\section{HYDRO + HEAVY-QUARK MODEL}

\section{A. Background QGP fluid}

The hydrodynamics has been quite successful in the description of collective flow phenomena in heavy ion collisions at RHIC. Since the hydrodynamics gives space-time evolution of temperature and flow velocity of the fluid so that the local rest frame of fluid is well-defined. Then, the Langevin equation in the previous section formulated in the local rest frame of the fluid is applicable directly.

Let us first summarize the relativistic hydrodynamic model [2, 19, 20, 21] whose basic equation reads

$$
\partial_{\mu} T^{\mu \nu}=0 .
$$

Here $T^{\mu \nu}$ is energy-momentum tensor. For strongly interacting matter with zero viscosity, $T^{\mu \nu}$ becomes

$$
T^{\mu \nu}=(e+P) u^{\mu} u^{\nu}-P g^{\mu \nu},
$$

where $e, P$, and $u^{\mu}$ are energy density, pressure, and four fluid velocity, respectively. The baryon chemical potential is neglected, because it is small near mid-rapidity at RHIC energies. We solve Eq. (14) in the Bjorken coordinates $\left(\tau, x, y, \eta_{s}\right)$, where $\tau=\sqrt{t^{2}-z^{2}}$ and $\eta_{s}=\frac{1}{2} \ln [(t+z) /(t-z)]$ are proper time and space-time rapidity, respectively.

In the high temperature $\left(T>T_{c}=170 \mathrm{MeV}\right)$ QGP phase, we employ the bag equation of state (EOS) for massless partons ( $u, d, s$ quarks and gluons) with $B^{1 / 4}=$ $247.19 \mathrm{MeV}$. Here the bag constant is tuned to have transition to the hadron resonance gas at $T_{c}$. In the hadron phase $\left(T<T_{c}=170 \mathrm{MeV}\right)$, a resonance gas of hadrons with the mass up to $\Delta(1232)$ is employed [21]. Volume fraction of QGP $f_{\mathrm{QGP}}$ in the mixed phase is

$$
f_{\mathrm{QGP}}=\frac{e-e_{\mathrm{had}}}{e_{\mathrm{QGP}}-e_{\mathrm{had}}},
$$

where $e_{\mathrm{QGP}}\left(e_{\mathrm{had}}\right)$ is the maximum (minimum) value of the energy density in the mixed phase. Later we will utilize $f_{\mathrm{QGP}}$ to define the effective lifetime of QGP and the freezeout condition for the heavy quarks.

Hot QGP with local thermalization is assumed to be produced at $\tau_{0}=0.6 \mathrm{fm}$. The entropy density distribution at $\tau_{0}$ in the mid-rapidity is taken to be proportional to a linear combination of the number densities 
of participants and binary collisions in the transverse plane [19]. For the initial condition of the flow velocity, Bjorken's scaling solution, $u_{x}\left(\tau_{0}\right)=u_{y}\left(\tau_{0}\right)=0$ and $u_{z}\left(\tau_{0}\right)=\sinh \eta_{s}$, is employed [22]. With these initial conditions, the hydrodynamic model can well reproduce the experimental data of charged particles at RHIC [2].

The space-time evolution of the QGP fluid obtained as above has been exploited for a quantitative study of hard and rare probes such as azimuthal jet anisotropy, nuclear modification factor of identified hadrons, disappearance of back-to-back jet correlation, $J / \psi$ suppression, and direct photon emission [23].

\section{B. Heavy quark diffusion in quark-gluon fluid}

We solve the Langevin equation (11) and (2) with the drag coefficient $\Gamma$ given by Eq. (12) in the local rest frame of the fluid element. The dimensionless parameter $\gamma$ is inversely proportional to the relaxation time $\tau_{\mathrm{Q}}$ of a heavy quark as

$$
\tau_{\mathrm{Q}}=\frac{1}{\Gamma}=\frac{M_{Q}}{\gamma T^{2}}
$$

The $\tau_{\mathrm{Q}}$ is listed in Table 凹(a) for three typical values, $\gamma=0.3$ (weak coupling), $\gamma=1.0$ (intermediate coupling), and $\gamma=3.0$ (strong coupling). The characteristic temperature felt by the heavy quark during the spacetime history in the QGP fluid is taken to be $210 \mathrm{MeV}$ (as for the reasoning of this number, see Sec. IV A 1).

Let us now introduce an effective lifetime of QGP, $\tau_{\mathrm{QGP}}$, by the following definition: At $\tau=\tau_{\mathrm{QGP}}$, the QGP fraction $f_{\mathrm{QGP}}$ in Eq. (16) at $x=y=z=0$ reaches to $f_{0}$, which takes a value between 0 and 1 . For $f_{0}=0$, the effective lifetime is defined as the time when QGP disappears completely, while $f_{0}=1$ corresponds to the time when hadronic phase starts to appear. The effective lifetime of QGP is listed in Table I(b) for two different impact parameters and for three different values of $f_{0}$.

From the comparison of $\tau_{\mathrm{Q}}$ and $\tau_{\mathrm{QGP}}$ in Table $\square$ one finds that the initial momentum distributions of charm and bottom quarks will be changed by QGP only slightly for the weak drag force $(\gamma=0.30)$. On the other hand, for the strong drag force $(\gamma=3.0)$, both charm and bottom quarks are affected by QGP and their momentum distributions would be modified substantially.

\section{Initial distribution of heavy quarks}

On the initial hypersurface $\tau_{0}=0.6 \mathrm{fm}$, initial transverse positions of heavy quarks are distributed according to the overlap function of two nuclei $\mathrm{A}$ and $\mathrm{B}$ in the (a)

\begin{tabular}{c|ccc}
\hline \hline$\gamma$ & 0.30 & 1.0 & 3.0 \\
\hline$\tau_{\mathrm{c}}[\mathrm{fm}]$ & 22 & 6.7 & 2.2 \\
$\tau_{\mathrm{b}}[\mathrm{fm}]$ & 72 & 21 & 7.2 \\
\hline
\end{tabular}

(b)

\begin{tabular}{|c|c|c|c|}
\hline$f_{0}$ & 0 & 0.5 & 1 \\
\hline$\tau_{\mathrm{QGP}}^{b=3.1 \mathrm{fm}} \quad[\mathrm{fm}]$ & 9.8 & 5.9 & 4.5 \\
\hline$\tau_{\mathrm{QGP}}^{b=5.5 \mathrm{fm}} \quad[\mathrm{fm}]$ & 8.7 & 5.2 & 4.0 \\
\hline
\end{tabular}

TABLE I: (a) Relaxation times of charm and bottom quarks for $\gamma=0.3,1.0$, and 3.0 at $T=210 \mathrm{MeV}$. (b) Lifetimes of QGP for different centralities and freezeout conditions. In (a) $M_{\mathrm{c}}$ and $M_{\mathrm{b}}$ are chosen to be $1.5 \mathrm{GeV}$ and $4.8 \mathrm{GeV}$, respectively. In (b) we adopt two characteristic impact parameters $b=3.1$ and $5.5 \mathrm{fm}$.

transverse plane $T_{\mathrm{AB}}(x, y)$ :

$$
\begin{aligned}
T_{\mathrm{AB}}(x, y) & =T_{\mathrm{A}}\left(x+\frac{b}{2}, y\right) T_{\mathrm{B}}\left(x-\frac{b}{2}, y\right), \\
T_{\mathrm{A}(\mathrm{B})}(x, y) & =\int d z \rho_{\mathrm{A}(\mathrm{B})}(x, y, z),
\end{aligned}
$$

where $\rho_{\mathrm{A}(\mathrm{B})}$ is the Woods-Saxon parametrization of nuclear density. The heavy quarks are assumed to stream freely in the longitudinal direction for $0<\tau<\tau_{0}$ and acquire the momentum rapidity $y_{p}=\eta_{s}$. Thus the initial heavy quark distribution in the phase space reads

$$
\frac{d N}{d^{3} p d^{2} x_{\perp} \tau_{0} d \eta_{s}}=\frac{d \sigma_{\mathrm{pp}}^{\mathrm{HQ}}}{d^{3} p} T_{\mathrm{AB}}(x, y) \frac{\delta\left(\eta_{s}-y_{p}\right)}{\tau_{0}} .
$$

The initial momentum spectrum of heavy quarks $d \sigma_{\mathrm{pp}}^{\mathrm{HQ}} / d^{3} p$ in $p+p$ collisions is calculated by perturbative QCD to leading order (LO) utilizing the event generator PYTHIA 6.4 [13].

In Fig. 1 the initial distribution of heavy quarks in the transverse plane and that in the momentum space at mid-rapidity $\left(\left|y_{p}\right| \leq 1\right)$ are shown. The momentum distribution is normalized to the invariant cross section in $p+p$ collisions. Note that the initial momentum distribution of the charm quark has a steeper slope at high $p_{\mathrm{T}}$ than that of the bottom quark. Nuclear effects such as shadowing and Cronin effect are not considered for simplicity.

In Fig. 2(a), we show the differential cross section of electrons from heavy quarks in $p+p$ collisions obtained by PYTHIA (the LO perturbative QCD). Theoretical cross section underestimates the experimental value by a factor 5-10 as shown in Fig. 2(b). The discrepancy is known to become smaller by taking into account higher orders beyond LO [24, 25]. Since we are mainly interested in the the "shape" of the $p_{\mathrm{T}}$ distribution above $3 \mathrm{GeV}$ in this paper, we adopt the LO result for simplicity in spite of the problem of absolute magnitude in the LO calculation.

\section{Simulation of the Brownian motion}

The Langevin equation of a heavy quark is defined in the local rest frame of QGP. The information of local 
(a) HQ configuration
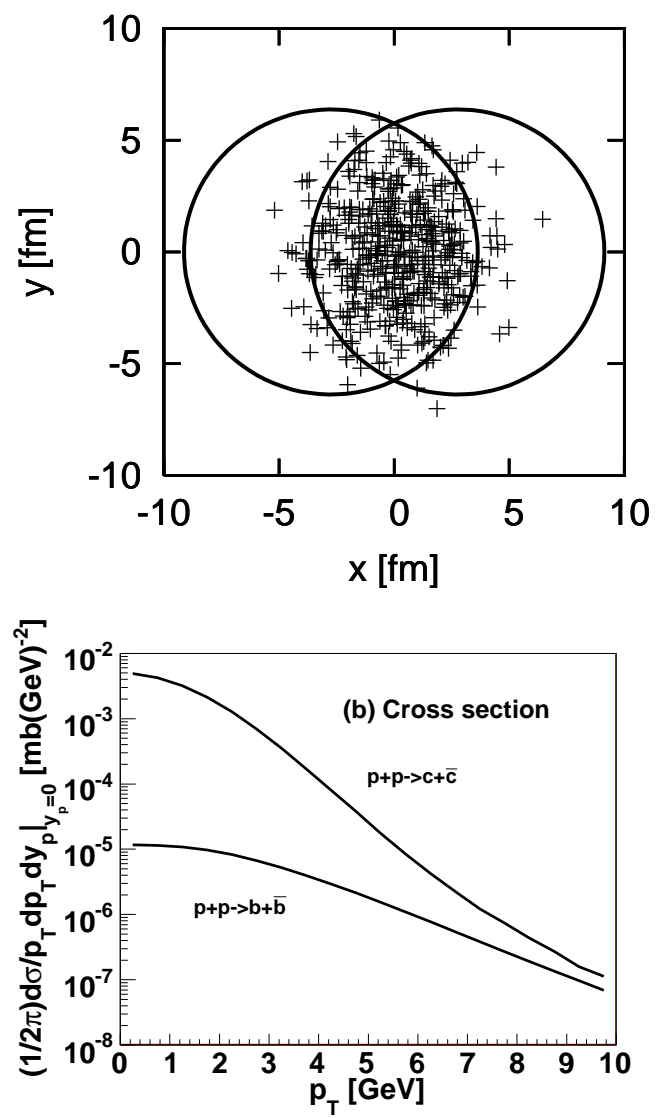

FIG. 1: (a) A sample of 500 heavy quarks at the initial in the transverse plane, for the $\mathrm{Au}+\mathrm{Au}$ collision with impact parameter 5.5 fm. (b) Invariant cross sections of charm and bottom production in $p+p$ collisions in mid-rapidity $\left(\left|y_{p}\right| \leq 1.0\right)$, which is proportional to the initial momentum distribution.

flow velocity and local temperature at the position of the heavy quark is supplied from the relativistic hydrodynamics. The algorithm of such Langevin simulation is summarized as follows.

(i) Start from a sample of heavy quark at a position and a momentum according to the initial phase space distribution given in Eq. (19).

(ii) Given the phase space location $\left(p^{\mu}, x^{\mu}\right)$ in the laboratory frame, obtain the information of the local flow velocity $u^{\mu}(x)$ and local temperature $T(x)$ from the output of the hydrodynamic simulation.

(iii-a) Coordinate step: Make one discrete step for the heavy quark in the configuration space according to Eq. (11) by using discrete proper-time step $\Delta s=$ $(M / E) \Delta t$ :

$$
\Delta x^{\nu}(s)=\frac{p^{\nu}}{M} \Delta s
$$
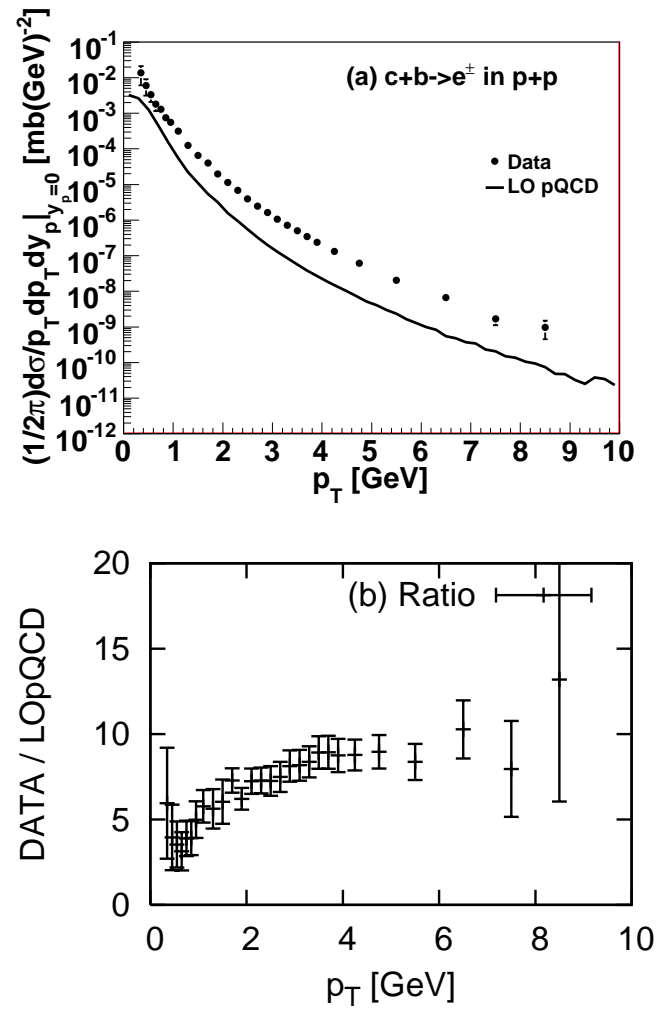

FIG. 2: (a) Experimental cross section for electron production in $p+p$ collision at mid-rapidity 24] and the leading order pQCD result by PYTHIA. (b) The ratio of the experimental data and the LO result. Theoretical calculations are performed at $\left|y_{p}\right| \leq 0.35$ and then properly normalized to obtain the cross section.

(iii-b) Momentum step: Move to the rest frame of the fluid element by the Lorentz transformation, $p \rightarrow k$. Make one discrete step for the heavy quark in momentum according to Eq. (2) using $\Delta s$ :

$$
\begin{aligned}
\Delta \vec{k}(s) & =-\gamma \frac{T^{2} E(k)}{M^{2}} \vec{k} \Delta s+\vec{\xi}(s), \\
\left\langle\xi_{i}(s) \xi_{j}\left(s^{\prime}\right)\right\rangle & =\delta_{i j} \delta_{s s^{\prime}} \frac{2 \gamma T^{3}}{M^{2}} E(E+T) \Delta s .
\end{aligned}
$$

Then, move back to the laboratory frame by inverse Lorentz transformation $\left(k+\Delta k \rightarrow p^{\prime}\right)$.

(iv) Repeat the steps (ii) and (iii) until the volume fraction of QGP in the mixed phase $\left(f_{\mathrm{QGP}}\right)$ reaches $f_{0}$.

Several comments are in order here about this procedure.

- We use the proper-time step $\Delta s$ instead of the ordinary time step $\Delta t$ in our simulation, simply because the former is a Lorentz scalar and thus easy to handle in going back and forth between the laboratory frame and the fluid rest frame. We choose 
$\Delta s=0.01 \mathrm{fm}$ in our simulation, which is much shorter than the relaxation time of the drag force parameter adopted in this paper.

- Owing to the Itô discretization scheme, the momentum step in (iii-b) can be performed only by using the information of flow and temperature at the current position of the heavy quark in the phase space.

- It is not clear whether we should stop the heavy quark diffusion at the point when the mixed phase starts to appear or at the point when the mixed phase disappears. We consider this uncertainty as a systematic error and consider the three cases as shown in Table 【(b), namely $f_{0}=0,0.5$, and 1 .

\section{Freezeout and decay}

Once the local temperature around the charm(bottom) quark becomes lower than $T_{\mathrm{c}}$, it hadronizes into $D(B)$ mesons. Since we need to calculate single electron spectra from the heavy quarks, we focus on the following semileptonic decays: $D \rightarrow e$ for $D$ decay, $B \rightarrow e$ for primary $B$ decay, and $B \rightarrow D \rightarrow e$ for secondary $B$ decay. The hadronization of heavy quarks and the decay of heavy mesons are calculated by using PYTHIA 6.4 [13]. Since we employ independent fragmentation given by PYTHIA, the effect of quark recombination to form $D$ or $B$ mesons is not taken into account. Such simplification would be more reasonable for heavy quarks with higher transverse momentum. Therefore, it is the high $p_{\mathrm{T}}$ region (e.g. above $3 \mathrm{GeV}$ ) that is suitable to compare our results with the experimental data.

\section{Observables}

Medium modification factor $R_{\mathrm{AA}}$ for single electrons is defined by

$$
R_{\mathrm{AA}}\left(p_{\mathrm{T}}\right)=\frac{1}{N_{\mathrm{coll}}} \frac{d N_{\mathrm{A}+\mathrm{A}} / d p_{\mathrm{T}}}{d N_{p+p} / d p_{\mathrm{T}}}
$$

where $N_{\text {coll }}$ is the number of binary collisions calculated from the Glauber model. Since the initial heavy quark distribution is assumed to be without nuclear effects and to scale as $N_{\text {coll }}$ in our calculation, the deviation of $R_{\mathrm{AA}}$ from unity is solely attributed to the heavy quark diffusion in the hot medium. The elliptic flow for single electrons is defined by

$$
v_{2}\left(p_{\mathrm{T}}\right)=\frac{\int d \phi \frac{d^{2} N_{\mathrm{A}+\mathrm{A}}}{d p_{\mathrm{T}} d \phi} \cos 2 \phi}{\int d \phi \frac{d^{2} N_{\mathrm{A}+\mathrm{A}}}{d p_{\mathrm{T}} d \phi}}=\langle\cos 2 \phi\rangle .
$$

This quantity indicates how much momentum anisotropy around the collision axis is given to the heavy quarks from the background medium.

\section{NUMERICAL RESULTS}

Before showing the numerical results in detail, let us first summarize the basic parameters of our simulation. (1) The dimensionless drag coefficient $\gamma$ is a parameter to control the diffusion of heavy quarks in QGP. We take three characteristic values, $\gamma=0.3,1.0$, and 3.0 corresponding to weak, intermediate, and strong coupling, respectively. (2) The impact parameter $b$ controls the volume and the lifetime of QGP. Thus it affects indirectly the heavy quark spectra at their freezeout and the single electron spectra. In all of the figures below except for Fig. 9 $b$ is taken to be $5.5 \mathrm{fm}$ (10-20\% centrality). (3) The criterion of stopping the heavy quark diffusion in the mixed phase is given by $f_{0}$ which takes a value between 0 and 1 . Its dependence on the final results is considered to be a systematic error of our calculation. In all of the figures except for Fig. 9, we show the results at the central value, $f_{0}=0.5$.

\section{A. Heavy quark spectra}

\section{Profile of heavy quark diffusion}

To estimate how long a heavy quark stays in the QGP region in terms of local fluid proper time, we define the "stay time" as

$$
\begin{aligned}
t_{\mathrm{S}} & \left.\equiv \sum_{\text {steps }} \Delta t\right|_{\mathrm{FRF}}=\left.\sum_{\text {steps }} \Delta s(E / M)\right|_{\mathrm{FRF}} \\
& =\left.\sum_{\text {steps }} \Delta s(p \cdot u / M)\right|_{\mathrm{LF}},
\end{aligned}
$$

where FRF and LF imply the fluid rest frame and laboratory frame, respectively. By averaging over the heavy quarks starting initially with $p_{T}^{\text {in }}$ and ending in midrapidity $\left(\left|y_{p}\right| \leq 1.0\right)$ at their freezeout, we obtain the average stay time $\left\langle t_{\mathrm{S}}\right\rangle$.

Shown in Fig. 3 is the averaged stay time of heavy quarks as a function of their initial transverse momentum. The diffusion coefficient is taken to be $\gamma=0,0.3$, 1.0, 3.0, and 30.0. Here $\gamma=0$ corresponds to the free streaming. On the other hand, $\gamma=30.0$ corresponds to the extremely strong coupling where the relaxation times at typical temperature $210 \mathrm{MeV}$ are $0.22 \mathrm{fm}$ for charm and $0.72 \mathrm{fm}$ for bottom: The initial information on $p_{\mathrm{T}}$ is completely lost after a few fm of diffusion in this case.

The figure shows that, for heavy quarks with large initial velocity compared to the fluid velocity $\left(p_{\mathrm{T}}^{c, \text { in }}>\right.$ $1 \mathrm{GeV}, p_{\mathrm{T}}^{b, \text { in }}>3 \mathrm{GeV}$ ), the stay time becomes shorter for higher $p_{\mathrm{T}}$ because they get out of the medium in shorter times. Also, as the drag force becomes stronger, the stay time becomes longer as expected. As for the heavy quarks with small initial velocity $\left(p_{\mathrm{T}}^{c, \text { in }}<1 \mathrm{GeV}\right.$, $\left.p_{\mathrm{T}}^{b, \text { in }}<3 \mathrm{GeV}\right)$, the stronger the drag force, the shorter the stay time, since the drag force from the background fluid accelerates them more strongly. 

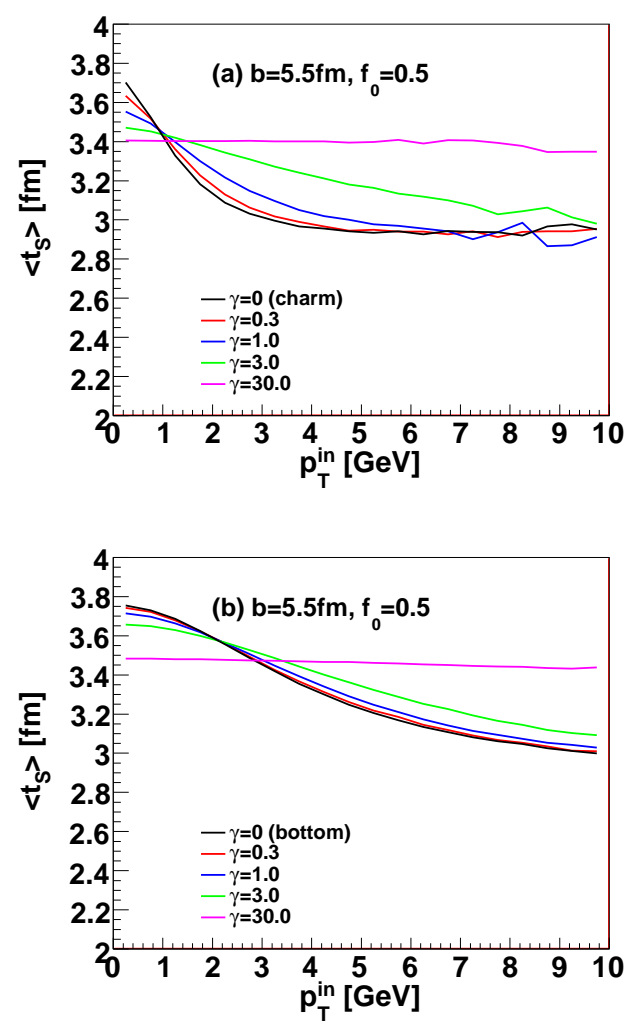

FIG. 3: (Color online) The averaged stay time $\left\langle t_{\mathrm{S}}\right\rangle$ of (a) charm quarks and (b) bottom quarks with the drag coefficient $\gamma=0$, $0.3,1.0,3.0$, and 30.0 at mid-rapidity $\left(\left|y_{p}\right| \leq 1.0\right)$. The impact parameter is chosen to be $5.5 \mathrm{fm}$ in $\mathrm{Au}+\mathrm{Au}$ collisions. For freezeout condition, $f_{0}=0.5$ is adopted.

Next we define the averaged temperature for the heavy quarks experienced during their stay in the QGP fluid:

$$
\left.\bar{T} \equiv\left(1 / t_{\mathrm{S}}\right) \sum_{\text {steps }} T(x) \Delta t\right|_{\mathrm{FRF}} .
$$

By averaging over the heavy quarks starting initially with $p_{\mathrm{T}}^{\mathrm{in}}$ and ending in mid-rapidity $\left(\left|y_{p}\right| \leq 1.0\right)$ at freezeout, we obtain the averaged temperature $\langle\bar{T}\rangle$ shown in Fig. 4

The figure shows that, for heavy quarks with large initial velocity compared to the fluid velocity $\left(p_{\mathrm{T}}^{c, \text { in }}>1\right.$ $\left.\mathrm{GeV}, p_{\mathrm{T}}^{b, \text { in }}>3 \mathrm{GeV}\right)$, the averaged temperature becomes higher for higher $p_{\mathrm{T}}^{\text {in }}$ because they feel only the initial high temperature region before getting out of QGP. Also, as the drag force becomes stronger, the stay time becomes longer and averaged temperature becomes smaller. As for the heavy quarks with small initial velocity $\left(p_{\mathrm{T}}^{c, \text { in }}<\right.$ $\left.1 \mathrm{GeV}, p_{\mathrm{T}}^{b, \text { in }}<3 \mathrm{GeV}\right)$, the stronger the drag force, the higher the averaged temperature, since they are strongly accelerated and quickly pass the low temperature region. It turns out that the average temperature lies between $200 \mathrm{MeV}$ and $220 \mathrm{MeV}$ in the wide range of $p_{\mathrm{T}}^{\text {in }}$ and $\gamma$; this is the reason why we adopted the typical temperature $210 \mathrm{MeV}$ in Sec. III]

Finally, let us define the the transverse momentum loss
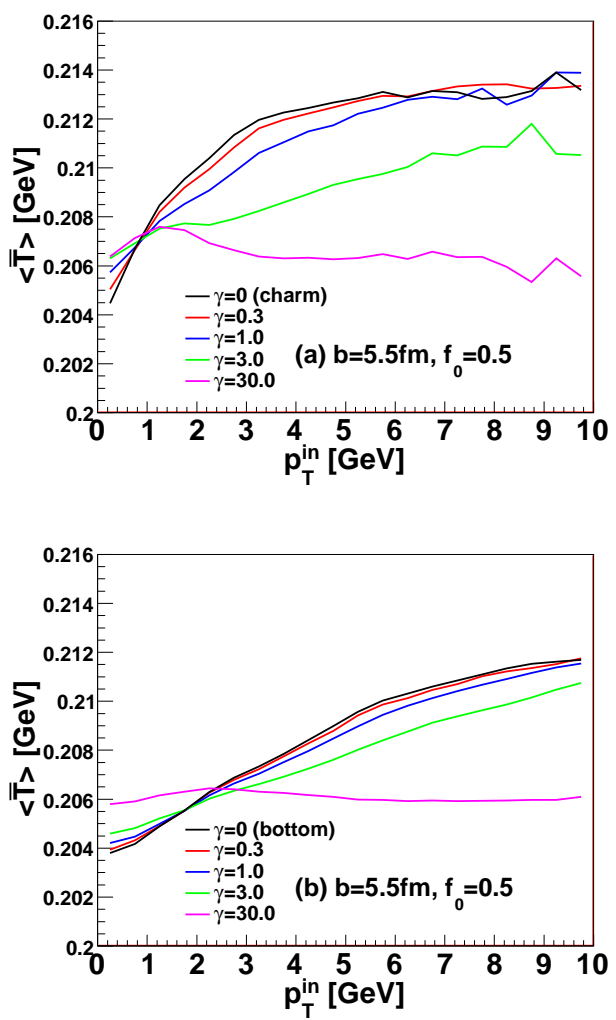

FIG. 4: (Color online) The averaged temperature $\langle\bar{T}\rangle$ of (a) charm quarks and (b) bottom quarks with the drag coefficient $\gamma=0$, $0.3,1.0,3.0$, and 30.0 in mid-rapidity $\left(\left|y_{p}\right| \leq 1.0\right)$. The collision geometry and the freezeout condition are the same with those in Fig. 3 The fluctuation in high $p_{\mathrm{T}}$ in (a) is due to statistical errors of our simulation.

(momentum loss for short):

$$
\Delta p_{\mathrm{T}}=p_{\mathrm{T}}^{\text {in }}-p_{\mathrm{T}}^{\text {out }},
$$

where $p_{\mathrm{T}}^{\text {out }}$ is the transverse momentum at the time of the freezeout of the heavy quark. By averaging over the heavy quarks starting initially with $p_{\mathrm{T}}^{\text {in }}$ and ending in mid-rapidity $\left(\left|y_{p}\right| \leq 1.0\right)$ at freezeout, we obtain the averaged momentum loss $\left\langle\Delta p_{\mathrm{T}}\right\rangle$ as shown in Fig. 囵

For heavy quarks with larger initial $p_{\mathrm{T}}^{\text {in }}$, the momentum loss per unit time (dynamical effect) is larger as seen in Eqs. (22) and (12) while the average stay time (kinematical effect) is shorter. Therefore, there are two competing effects in the net momentum loss: In Fig. 5 , we find that larger initial momentum leads to larger momentum loss, so that the dynamical effect wins over the kinematical effect. As for the dependence on drag coefficient, both dynamical and kinematical effects act additively for heavy quarks with large initial momentum $\left.\left(p_{\mathrm{T}}^{c \text {,in }}>1 \mathrm{GeV}, p_{\mathrm{T}}^{b, \text { in }}>1.5 \mathrm{GeV}\right)\right)$ and the momentum loss is enhanced by increasing $\gamma$. For the heavy quarks with small initial velocity $\left(p_{\mathrm{T}}^{c, \text { in }}<1 \mathrm{GeV}, p_{\mathrm{T}}^{b \text {,in }}<1.5\right.$ $\mathrm{GeV}$ ), these two effects compete but we find in Fig. 5 that the dynamical effect seems to win, namely that the 

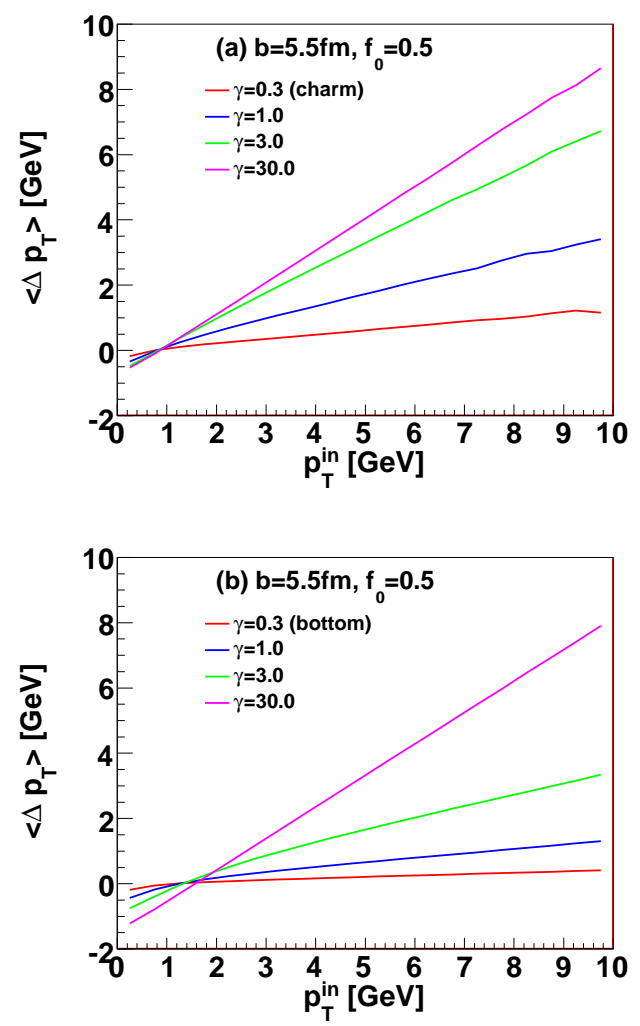

FIG. 5: (Color online) The averaged momentum loss $\left\langle\Delta p_{\mathrm{T}}\right\rangle$ of (a) charm quarks and (b) bottom quarks with drag coefficients $\gamma=$ $0.3,1.0,3.0$, and 30.0 in mid-rapidity $\left(\left|y_{p}\right| \leq 1.0\right)$. The collision geometry and the freezeout condition are the same with those in Fig. 3 .

stronger the drag force, the larger the momentum gain by the acceleration from the fluid. Note here that, for the extreme case $\gamma=30.0$, we have almost a linear increase of $\left\langle\Delta p_{\mathrm{T}}\right\rangle=p_{\mathrm{T}}^{\text {in }}-p_{\mathrm{T}}^{\text {out }}$ as a function of $p_{\mathrm{T}}^{\text {in }}$. This is simply because the heavy quarks are thermalized and $p_{\mathrm{T}}^{\text {out }}$ is almost independent of $p_{\mathrm{T}}^{\text {in }}$.

\section{Nuclear modification factor $R_{\mathrm{AA}}^{Q}$}

Let us define $R_{\mathrm{AA}}^{Q}(Q=c, b)$ for heavy quarks by replacing the number of electrons $N_{p+p}\left(N_{\mathrm{A}+\mathrm{A}}\right)$ in Eq. (23) by the number of heavy quarks at the freezeout $N_{p+p}^{Q}$ $\left(N_{\mathrm{A}+\mathrm{A}}^{Q}\right)$. This is a theoretical quantity not directly accessible in experiment, but it is useful to examine the behavior of heavy quarks without the kinematical complication due to their semileptonic decays to electrons.

Shown in Fig. 6 are $R_{\mathrm{AA}}^{Q}$ for charm and bottom in the mid-rapidity at impact parameter $5.5 \mathrm{fm}$ as a function of $p_{\mathrm{T}}^{\text {out }}$. There are two key factors which determine $R_{\mathrm{AA}}^{Q}$; the momentum loss of heavy quarks and the initial distribution of heavy quarks. Starting from the initial distribution, the high momentum quarks loose energy due to
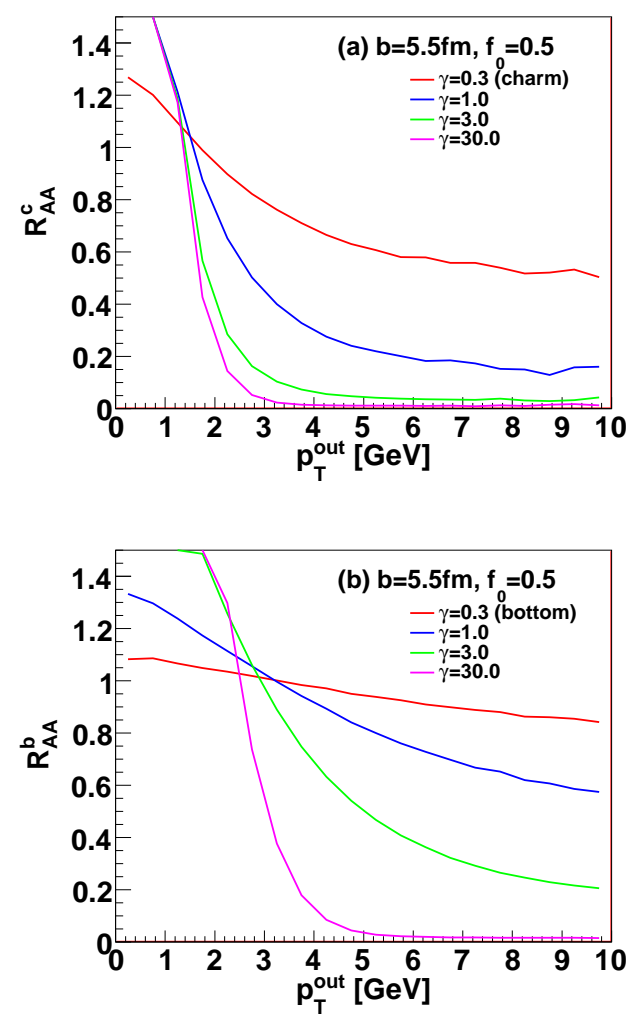

FIG. 6: (Color online) $R_{\mathrm{AA}}^{Q}$ of (a) charm quarks and (b) bottom quarks with drag coefficients $\gamma=0.3,1.0,3.0$, and 30.0 at midrapidity $\left(\left|y_{p}\right| \leq 1.0\right)$. For collision geometry, we choose the impact parameter $5.5 \mathrm{fm}$ in $\mathrm{Au}+\mathrm{Au}$ collisions. For freezeout condition, the $f_{0}=0.5$ is adopted.

drag force and are shifted to the low $p_{\mathrm{T}}^{\text {out }}$ region. Therefore, $R_{\mathrm{AA}}^{Q}$ is suppressed (enhanced) at high (low) $p_{\mathrm{T}}^{\text {out }}$. This tendency is prominent for large drag force as expected. Also, the suppression at high $p_{\mathrm{T}}^{\text {out }}$ is larger for the charm if we adopt the same $\gamma$. This is because the actual drag coefficient is $\gamma T^{2} / M_{Q}$ so that the quark with smaller mass is affected more by the drag force.

\section{Elliptic flow $v_{2}^{Q}$}

In Fig. (7) we show the elliptic flow for the heavy quark $v_{2}^{Q}(Q=c, b)$ at mid-rapidity at impact parameter $5.5 \mathrm{fm}$. It is clear that the charm and bottom quarks with any drag force at large $p_{\mathrm{T}}^{\text {out }}$ are less thermalized and thus they do not produce much momentum anisotropy. Note that the dominant contributions of heavy quarks with $\gamma=$ 30.0 at large $p_{\mathrm{T}}^{\text {out }}$ may be those that start near the surface of the QGP fireball and with large initial $p_{\mathrm{T}}^{\mathrm{in}}$, therefore they are not much thermalized because of the too short stay times. On the other hand, charm quarks with small $p_{\mathrm{T}}^{\text {out }}$ are thermalized for large drag force and develops $v_{2}^{Q}$ reflecting the flow of light particles. As for bottom quarks, they only have small momentum anisotropy with 

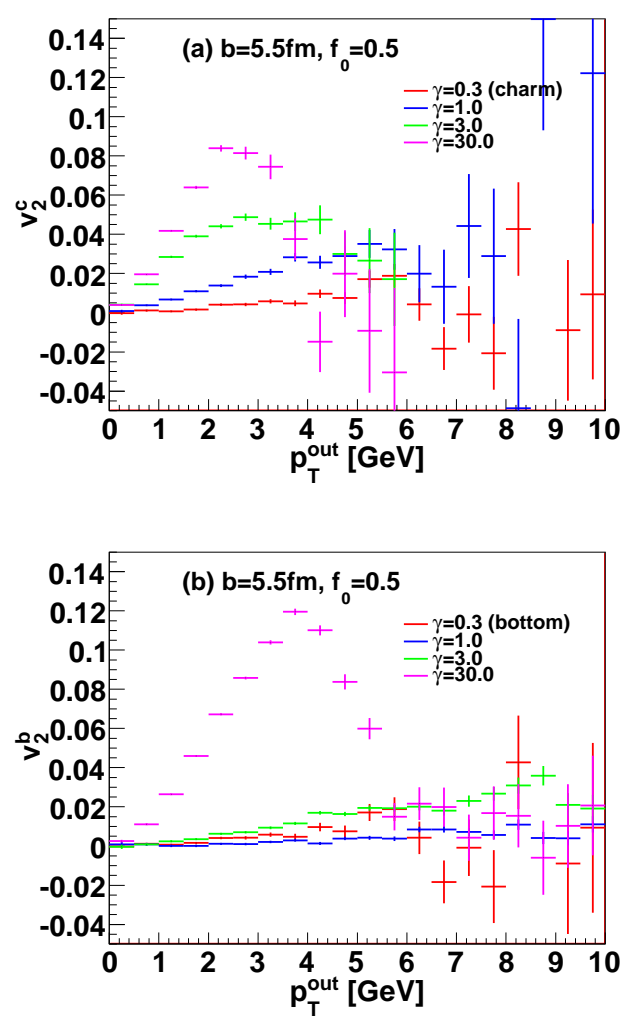

FIG. 7: (Color online) $v_{2}^{Q}$ of (a) charm quarks and (b) bottom quarks with drag coefficients $\gamma=0.3,1.0,3.0$, and 30.0 in midrapidity $\left(\left|y_{p}\right| \leq 1.0\right)$. The collision geometry and the freezeout condition are the same with those in Fig. 6 In (a), the statistical errors for $\gamma=3.0$ and 30.0 are so large at $p_{\mathrm{T}}^{\text {out }}>6 \mathrm{GeV}$ that we omit them.

all drag forces but $\gamma=30.0$ at small $p_{\mathrm{T}}^{\text {out }}$ because they are not enough thermalized.

\section{B. Electron spectra}

\section{Nuclear modification factor $R_{\mathrm{AA}}$}

Let us now examine the results of electrons and positrons (we call them just electrons for short) which are the decay products from $D$ and/or $B$ mesons. In Fig. $8(\mathrm{a}),(\mathrm{b})$, and (c), we show $R_{\mathrm{AA}}$ of electrons (a) from charm quarks, (b) from bottom quarks, and (c) from charm+bottom quarks. The dependence of $R_{\mathrm{AA}}$ on the drag coefficient $\gamma$ is understood easily: Larger drag coefficient gives larger energy loss and $R_{\mathrm{AA}}$ is suppressed. There is however one qualitative difference between $R_{\mathrm{AA}}^{Q}$ in Sec. IVA2 and $R_{\mathrm{AA}}$ in the low $p_{\mathrm{T}}$ region: $R_{\mathrm{AA}}^{Q}$ exceeds unity due to the shift of the high momentum quarks to low momentum quarks, while $R_{\mathrm{AA}}$ stays around unity at low momentum. This is understood by recognizing that the low $p_{\mathrm{T}}$ electrons come from wide range of heavy quarks with various freezeout momenta, so that low mo-
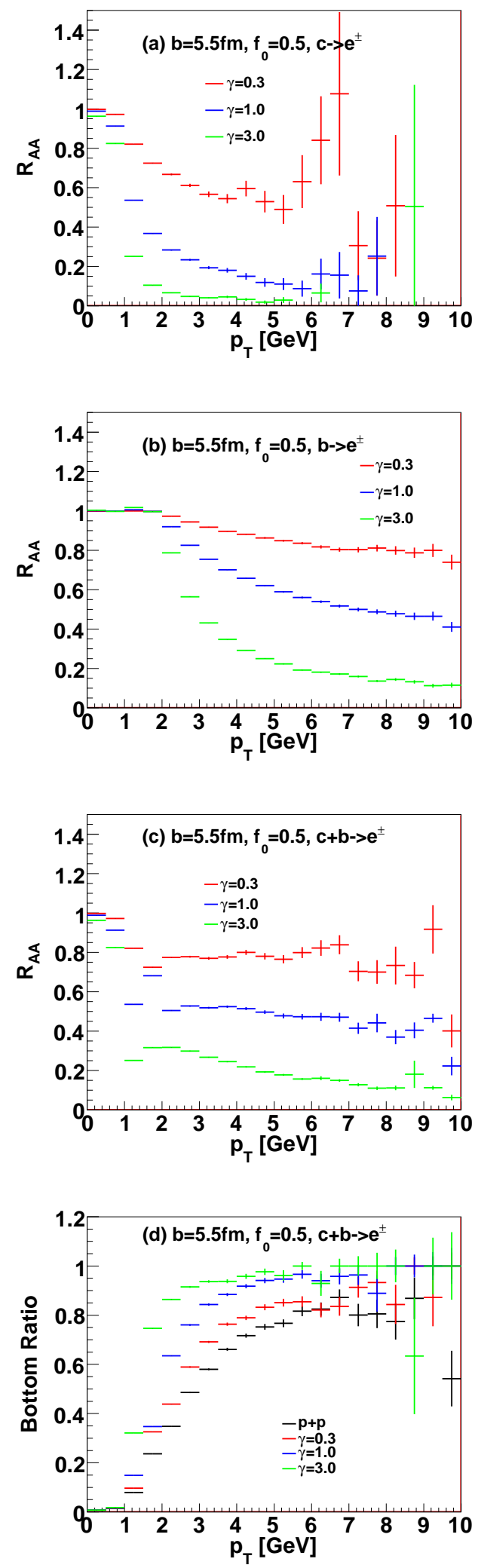

FIG. 8: (Color online) (a) $R_{\mathrm{AA}}$ of electrons from charm, (b) $R_{\mathrm{AA}}$ of electrons from bottom, (c) $R_{\mathrm{AA}}$ of electrons from both charm and bottom, and (d) the ratio of electrons from the bottom and the net electrons. All results are in mid-rapidity $\left(\left|y_{p}\right| \leq 0.35\right)$. The drag coefficient is taken to be $\gamma=0.3,1.0$, and 3.0. The impact parameter is taken to be $5.5 \mathrm{fm}$ in $\mathrm{Au}+\mathrm{Au}$ collisions. For freezeout condition, the $f_{0}=0.5$ is adopted. In (d), the result of $p+p$ collision calculated in the leading order pQCD by PYTHIA is also plotted. 

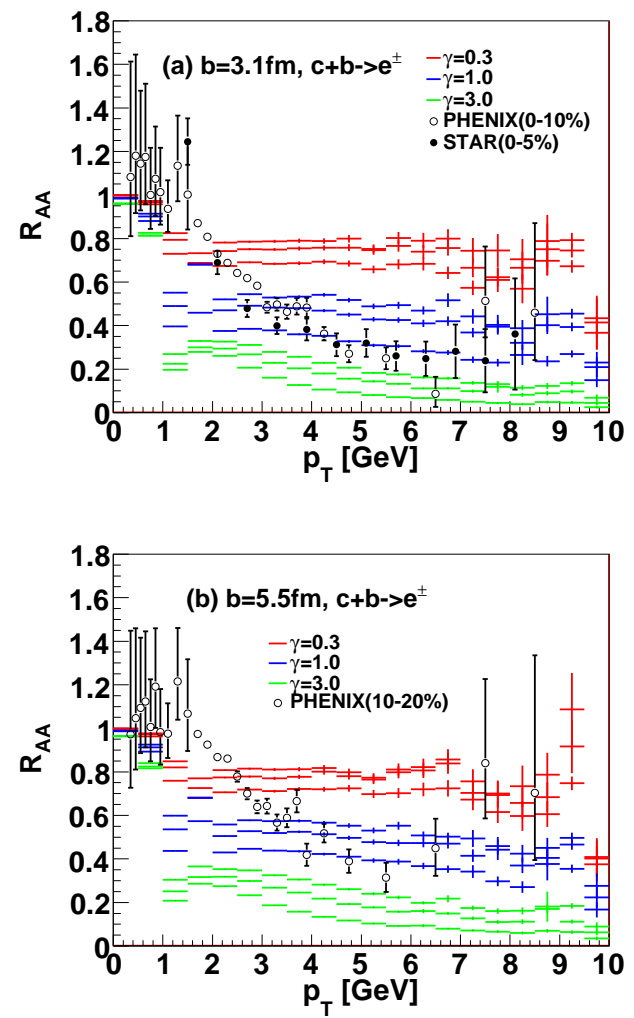

FIG. 9: (Color online) Comparison of $R_{\mathrm{AA}}$ in our hydro + heavyquark model with the experimental data [4, 5]. The Au+Au collision with the impact parameter (a) $3.1 \mathrm{fm}$ and (b) $5.5 \mathrm{fm}$, both in mid-rapidity, $\left|y_{p}\right| \leq 0.35$. The drag coefficient is chosen to be $\gamma=0.3,1.0$, and 3.0 indicated by different colors. The freezeout condition is taken to be $f_{0}=1.0,0.5$, and 0.0 which correspond to upper, middle, and lower points, respectively, within the same color. As for error bars in experimental data, we only plot the statistical errors [4, 5].

mentum electrons are not sensitive to the modification of the heavy quark spectrum due to diffusion. On the other hand, the electrons with high $p_{\mathrm{T}}$ originate mainly from high $p_{\mathrm{T}}$ heavy quarks and thus they are sensitive to the spectral change of heavy quarks.

In Fig. $8(d)$, the number of electrons from bottom divided by that from charm+bottom for $\mathrm{Au}+\mathrm{Au}$ collision is shown as a function of electron's $p_{\mathrm{T}}$ together with that for $p+p$ collision. In both $p+p$ and $\mathrm{A}+\mathrm{A}$, more than $50 \%$ of electrons come from the bottom for $p_{\mathrm{T}}>3 \mathrm{GeV}$. Furthermore, the ratio increases as the drag force becomes stronger. The kink structure of $R_{\mathrm{AA}}$ at $p_{\mathrm{T}} \sim 1-2 \mathrm{GeV}$ in Fig. 8 (c) is understood by the fact that the dominant contribution to the electrons changes rapidly from the charm to the bottom.

Finally we compare our numerical results with experimental data [4] in Fig. 9. Here we show two cases of impact parameters $3.1 \mathrm{fm}$ (0-10\% centrality) and $5.5 \mathrm{fm}$ (10-20\% centrality) at mid-rapidity. The systematic errors due to the freezeout condition of heavy quark are represented by the three plots with the same color. Re-

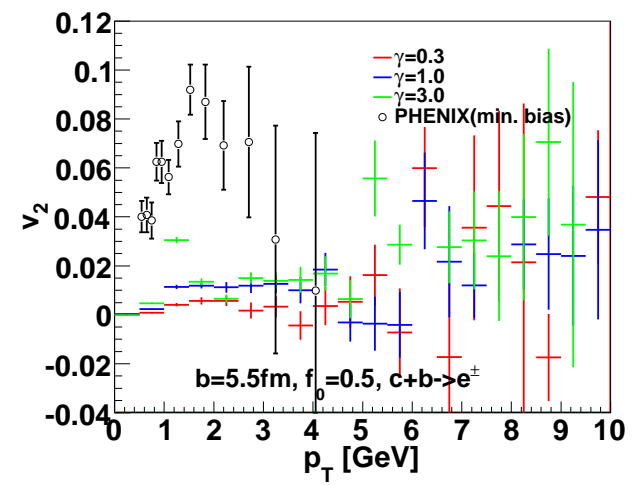

FIG. 10: (Color online) Comparison of $v_{2}$ in our hydro + heavyquark model with experimental data [4] in mid-rapidity $\left(\left|y_{p}\right| \leq\right.$ 0.35). Experimental data of $v_{2}$ is obtained in minimum bias analysis, while our theoretical values of $v_{2}$ are evaluated at impact parameter $5.5 \mathrm{fm}$ as a representative. The drag coefficient is chosen to be $\gamma=0.3,1.0$, and 3.0 and the freezeout condition is $f_{0}=0.5$. As for error bars in experimental data, we only plot the statistical errors [4].

call that the comparison of our results and experimental data is only reliable for $p_{\mathrm{T}}>3 \mathrm{GeV}$ as discussed in Sec. IIIB 1 and that bottom quarks are the dominant source of electrons in this region.

Although definite conclusion cannot be made from the present comparison, it is likely that the intermediate to large value of the drag coefficient $\gamma=1.0-3.0$ is favored especially for small impact parameter. This number is rather close to the value $\gamma=2.1 \pm 0.5$ predicted from the AdS/CFT correspondence (see Eq. (11)). We should remark, however, that the radiative energy loss [7, 18] and the relativistic diffusion via resonances combined with quark coalescence [17] would be legitimate alternatives to describe the data, so that further systematic comparison of the data and theoretical calculations is called for.

\section{Elliptic flow $v_{2}$}

We show our theoretical $v_{2}$ of electrons in Fig. 10 as a function of $p_{\mathrm{T}}$ together with the experimental data [4]. Our $v_{2}$ does not depend much on the strength of the drag force for $p_{\mathrm{T}}>3 \mathrm{GeV}$ and stays small. Due to the poor statistic of both our simulation and the experimental data in the relevant region, it is not clear whether theory and experiment are consistent with each other or not. Although it is still preliminary, recent PHENIX data show large $v_{2}=0.05-0.1$ with small errors for $3<p_{\mathrm{T}}<5$ $\mathrm{GeV}$ at collisions with corresponding centrality [26].

\section{SUMMARY AND CONCLUDING REMARKS}

In this paper, we have examined the diffusion of heavy quarks in the dynamical QGP fluid on the ba- 
sis of the relativistic Langevin equation combined with the relativistic hydrodynamics. We establish a generalized fluctuation-dissipation relation in Itô discretization scheme, Eq. (7), which relates the diffusion constant $D(p)$ and the drag coefficient $\Gamma(p)$ for the relativistic Brownian particle. Then we parametrized the drag coefficient motivated by the formula from the AdS/CFT correspondence for strongly coupled plasma, $\Gamma \equiv \gamma T^{2} / M$ with the dimensionless coefficient $\gamma$ as a parameter. The spacetime evolution of the QGP fluid composed of light quarks and gluons is treated by the full $(3+1)$-dimensional relativistic hydrodynamics for the perfect fluid.

By solving the Langevin equation for heavy quarks under the influence of the QGP fluid, we obtain the space-time history of the diffusion process of charm and bottom in the realistic situation of the relativistic heavy-ion collisions. The initial space-time distributions of charm/bottom are given by the event generator PYTHIA. The hadronization and the semileptonic decays of charm/bottom after they leave from the QGP region are treated by independent fragmentation and decay. $\mathrm{Nu}-$ clear effects for initial charm/bottom distributions and the quark recombination/coalescence in hadronization of heavy quarks, which would be important for low $p_{\mathrm{T}}<3$ $\mathrm{GeV}$ region of the final electron spectrum, are neglected for simplicity in this paper.

Since we have the space-time history of the charm/bottom during their diffusion, we have looked at the average stay time of heavy quarks in QGP $\left\langle t_{\mathrm{S}}\right\rangle$, the average temperature felt by heavy quarks in QGP $\langle\bar{T}\rangle$, and the average momentum loss $\langle\Delta p\rangle$ during the diffusion. We have also looked at the nuclear modification factor $R_{\mathrm{AA}}^{Q}$ and the elliptic flow $v_{2}^{Q}$ of heavy quarks as a function of the transverse momentum of the heavy quarks at their freezeout $p_{\mathrm{T}}^{\text {out }}$. The results indicate that, for sufficiently large values of $p_{\mathrm{T}}^{\text {out }}>3 \mathrm{GeV}$, there is a sizable suppression of $R_{\mathrm{AA}}^{Q}$ for large drag coefficient, while one can see only a significant effect in $v_{2}^{Q}$ only for $p_{\mathrm{T}}^{\text {out }}<3$ $\mathrm{GeV}$ which is not the region one can rely on our calculation.

Then we have compared our calculations of $R_{\mathrm{AA}}$ and the elliptic flow $v_{2}$ for single electron with the RHIC data. First of all, the momentum distribution of the electrons do not necessary reflect the shape of the momentum distribution of the heavy quarks at their freezeout due to decay kinematics. Also, the net electrons with $p_{\mathrm{T}}>3$ $\mathrm{GeV}$ are dominated by those from bottom quarks. A rough comparison of $R_{\mathrm{AA}}$ for $p_{\mathrm{T}}>3 \mathrm{GeV}$ suggests that the drag coefficient could be as large as $\gamma=1.0-3.0$. On the other hand, we are unable to extract useful information from $v_{2}$ for $p_{\mathrm{T}}>3 \mathrm{GeV}$ because of the lack of statistics in both experiment and simulations. The value of $\gamma=1.0-3.0$ is consistent with that predicted by AdS/CFT approach for strongly interacting plasma $(\gamma=2.1 \pm 0.5)$, although we could not exclude other descriptions of heavy quarks in QGP such as radiative stopping [7, 18] and the resonance scattering model [17]. High precision experimental data at RHIC and LHC for electrons from charm and bottom identified separately are highly desirable. Also the correlation of the transverse momenta of a heavy quark and a heavy anti-quark (and the associated electron-positron or $D-\bar{D}$ correlation [27, 28]) could be a good observable to make detailed comparison of the theories and experiments.

Before closing, we remark possible improvements of our approach to treat the region $p_{\mathrm{T}}<3 \mathrm{GeV}$ in a more reliable way: (i) Initial heavy quark distributions beyond LO need to be considered for better control of their absolute magnitude, the $p_{\mathrm{T}}$ shape, and the charm/bottom ratio, (ii) nuclear effects on the initial charm/bottom distribution need to be examined, and (iii) the hadronization of charm/bottom due to quark recombination processes needs to be taken into account.

\section{Acknowledgments}

Part of this work was carried out during Y. Akamatsu's visit to European Centre for Theoretical Studies in $\mathrm{Nu}$ clear Physics and Related Areas (ECT*). Y. A. thanks ECT $^{*}$ and J.P. Blaizot for their kind hospitality. The authors thank Tetsuo Matsui for fruitful discussions. Y. A. is supported by JSPS fellowships for Young Scientists. T. Hatsuda and T. Hirano are supported in part by the Grants-in-Aid of the Japanese Ministry of Education, Culture, Sports, Science, and Technology (Nos. 18540253, 19740130).

\section{APPENDIX A: RELATIVISTIC KRAMERS EQUATION}

In this Appendix, we derive the partial differential equation of $P(\vec{p}, \vec{x}, t)$ or the Kramers equation using the Itô (pre-point) discretization scheme. We give here the general form of the relativistic Langevin equation as

$$
\begin{aligned}
\Delta \vec{x}(t) & =\frac{p(\tilde{t})}{E(p(\tilde{t}))} \Delta t \\
\Delta \vec{p}(t) & =-A(p(\tilde{t})) \vec{p}(\tilde{t}) \Delta t+\sqrt{B(p(\tilde{t}))} \vec{\eta}(t) \Delta t, \\
W[\vec{\eta}(t)] d^{3} \eta(t) & =C \cdot \exp \left[-\frac{\Delta t}{2} \vec{\eta}(t)^{2}\right] d^{3} \eta(t), \\
\left\langle\eta_{i}(t) \eta_{j}\left(t^{\prime}\right)\right\rangle & =\frac{\delta_{i j} \delta_{t t^{\prime}}}{\Delta t},
\end{aligned}
$$

where $E(p)=\sqrt{\vec{p}^{2}+M^{2}}$ with $M$ being the mass of the Brownian particle. Here $\tilde{t} \equiv t$ corresponds to the Itô discretization and $\tilde{t} \equiv t+\Delta t$ corresponds to the HanggiKlimontovic discretization [15]. Also $A(p), B(p)$, and $\vec{\eta}(t)$ in the Itô discretization correspond to $\Gamma(p), D(p)$, and $\vec{\xi}(t) /(\sqrt{D(p)} \Delta t)$ in the text, respectively. Since the Langevin equation is based on Markovian process, one needs information only at time $t^{\prime}$ in order to know the 
probability at later time $t$ :

$$
\begin{aligned}
& P\left(\vec{p}, \vec{x}, t \mid \vec{p}_{0}, \vec{x}_{0}, t_{0}\right) \\
& =\int d^{3} p^{\prime} d^{3} x^{\prime} P\left(\vec{p}, \vec{x}, t \mid \vec{p}^{\prime}, \vec{x}^{\prime}, t^{\prime}\right) P\left(\vec{p}^{\prime}, \vec{x}^{\prime}, t^{\prime} \mid \vec{p}_{0}, \vec{x}_{0}, t_{0}\right)
\end{aligned}
$$

where $P\left(X, t \mid X_{0}, t_{0}\right)(X=\{\vec{p}, \vec{x}\})$ represents the conditional distribution function with a fixed initial condition $X_{0}$ at time $t_{0}$. In order to derive the partial differential equation, we have to calculate $P\left(X, t+\Delta t \mid X^{\prime}, t\right)$ from the Langevin equation. From the definition of $P\left(X, t+\Delta t \mid X^{\prime}, t\right)$,

$$
\begin{aligned}
& \left.P\left(X, t+\Delta t \mid X^{\prime}, t\right) \equiv\langle\delta(X-X(t+\Delta t))\rangle\right|_{t, X^{\prime}} \\
& \quad=\left\langle\delta\left[X-X^{\prime}-\Delta X(\eta(t), t)\right]\right\rangle \\
& \quad=\sum_{m=0}^{\infty}\left\langle[-\Delta X(\eta(t), t)]^{m}\right\rangle \frac{1}{m !} \partial_{X}^{m} \delta\left(X-X^{\prime}\right), \\
& \langle Y(\eta(t), t)\rangle \equiv \int d^{3} \eta(t) W[\eta(t)] Y(\eta(t), t) .
\end{aligned}
$$

Here $\left.\langle\cdots\rangle\right|_{t, X^{\prime}}$ in the first line of Eq. (A33) represents the conditional probability with the fixed initial condition $X(t)=X^{\prime}$. Note that in the last line of Eq. A3 , the average is expressed by the variables at time $t$. Inserting Eq. (A33) into Eq. (A2), we obtain

$$
\begin{aligned}
& P\left(X, t+\Delta t \mid X_{0}, t_{0}\right) \\
& =\int d X^{\prime} P\left(X, t+\Delta t \mid X^{\prime}, t\right) P\left(X^{\prime}, t \mid X_{0}, t_{0}\right) \\
& =\int d X^{\prime}\left[\delta\left(X-X^{\prime}\right)+\sum_{m=1}^{\infty}\left\langle[-\Delta X(\eta(t), t)]^{m}\right\rangle\right. \\
& =P\left(X, t \mid X_{0}, t_{0}\right) \\
& \left.\quad+\sum_{m=1}^{\infty} \frac{1}{m !} \partial_{X}^{m} \delta\left(X-X^{\prime}\right)\right] \cdot P\left(X^{\prime}, t \mid X_{0}, t_{0}\right) \\
& =P\left(X, t \mid X_{0}, t_{0}\right)+\Delta t \partial_{t} P\left(X, t \mid X_{0}, t_{0}\right) .
\end{aligned}
$$

In the Itô discretization scheme, the relevant average values $\left\langle[\Delta X(\eta(t), t)]^{m}\right\rangle$ are

$$
\begin{aligned}
\langle\Delta \vec{x}(t)\rangle & =\frac{\vec{p}(t)}{E(p(t))} \Delta t, \\
\langle\Delta \vec{p}(t)\rangle & =-A(p(t)) \vec{p}(t) \Delta t, \\
\left\langle\Delta p_{i}(t) \Delta p_{j}(t)\right\rangle & =B(p(t)) \delta_{i j} \Delta t,
\end{aligned}
$$

and the others are in higher order in $\Delta t$.

From Eqs. A4 and (A5), the resulting relativistic Kramers equation reads

$$
\begin{aligned}
& \left(\frac{\partial}{\partial t}+\frac{\vec{p}}{E} \frac{\partial}{\partial \vec{x}}\right) P(\vec{p}, \vec{x}, t) \\
& =\frac{\partial}{\partial \vec{p}}\left(A(p) \vec{p}+\frac{1}{2} \frac{\partial}{\partial \vec{p}} B(p)\right) P(\vec{p}, \vec{x}, t) .
\end{aligned}
$$

[1] K. Yagi, T. Hatsuda and Y. Miake, Quark - Gluon Plasma (Cambridge Univ. Press, Cambridge, 2005). Proceedings of Quark Matter 2006, J. Phys. G 34 (2007).

[2] T. Hirano, N. van der Kolk and A. Bilandzic, arXiv:0808.2684 [nucl-th].

[3] Reviewed in, A. Vitev, arXiv:0806.0003 [hep-ph].

[4] A. Adare et al. (PHENIX Collaboration), Phys. Rev. Lett. 98, 172301 (2007).

[5] B.I. Abelev et al. (STAR Collaboration), Phys. Rev. Lett. 98, 192301 (2007).

[6] Reviewed in M. Djordjevic, J. Phys. G 32, S333 (2006).

[7] S. Wicks, W. Horowitz, M. Djordjevic and M. Gyulassy, Nucl. Phys. A784, 426 (2007).

[8] S. Caron-Huot and G.D. Moore, Phys. Rev. Lett. 100,
052301 (2008); JHEP 0802, 081 (2008).

[9] S.S .Gubser, Phys. Rev. D 74, 126005 (2006).

[10] J. Casalderrey-Solana and D. Teaney, Phys. Rev. D 74, 085012 (2006).

[11] C.P. Herzog, A. Karch, P. Kovtun, C. Kozcaz and L.G. Yaffe, JHEP 07, 013 (2006).

[12] S.S. Gubser, Phys. Rev. D 76, 126003 (2007).

[13] T. Sjostrand, S. Mrenna and P. Skands, JHEP 0605, 026 (2006).

[14] P. Langevin, C. R. Acad. Sci. Paris 146, 530 (1908). N.G. Van Kampen, Stochastic Processes in Physics and Chemistry (North-Holland, Amsterdam, 1981).

[15] F. Debbasch, K. Mallick and J.P. Rivet, J. of Stat. Phys. 88, 945 (1997). F. Debbasch and J.P. Rivet, J. of Stat. 
Phys. 90, 1179 (1998)

See also, C. Chevalier and F. Debbasch, J. Math. Phys. 49, 043303 (2008) and references therein.

[16] G.D. Moore and D. Teaney, Phys. Rev. C 71, 064904 (2005).

[17] H. van Hees and R. Rapp, Phys. Rev. C 71, 034907 (2005).

See also, R. Rapp and H. van Hees, arXiv:0803.0901 [hep$\mathrm{ph}]$ and references therein.

[18] N. Armesto, M. Cacciari, A. Dainese, C.A. Salgado and U.A. Wiedemann, Phys. Lett. B 637, 362 (2006).

[19] T.Hirano, U.Heinz, D.Kharzeev, R.Lacey and Y.Nara, Phys. Let. B636, 299 (2006).

[20] T. Hirano, Phys. Rev. C 65, 011901 (2002).

[21] T. Hirano and K. Tsuda, Phys. Rev. C 66, 054905 (2002).

[22] J.D. Bjorken, Phys. Rev. D 27, 140 (1983).

[23] T. Hirano and Y. Nara, Phys. Rev. C 68, 064902 (2003);
C 69, 034908 (2004); Phys. Rev. Lett. 91, 082301 (2003); M. Isse, T. Hirano, R. Mizukawa, A. Ohnishi, K. Yoshino, and Y. Nara, arXiv:nucl-th/0702068, T. Gunji, H. Hamagaki, T. Hatsuda, and T. Hirano, Phys. Rev. C 76, 051901(R) (2007); F.-M. Liu, T. Hirano, K. Werner, and Y. Zhu, arXiv:0807.4771 [hep-ph].

[24] A. Adare et al. (PHENIX Collaboration), Phys. Rev. Lett. 97, 252002 (2006)

[25] M. Cacciari, P. Nason and R. Vogt, Phys. Rev. Lett. 95, 122001 (2005).

[26] D. Hornback, talk at Quark Matter 2008.

[27] X. Zhu, M. Bleicher, S.L. Huang, K. Schweda, H. Stoecker, N. Xu and P. Zhuang, Phys. Lett. B 647, 366 (2007).

[28] X. Zhu, N. Xu and P. Zhuang, Phys. Rev. Lett. 100, $152301(2008)$ 